\title{
Prognostic impact of digoxin use for rate control of atrial fibrillation in patients $\geq 75$ years of age
}

\author{
Maria Teresa La Rovere, Egidio Traversi \\ Department of Cardiology, Istituti Clinici Scientifici Maugeri, IRCCS Montescano, Italy
}

\begin{abstract}
Digoxin use remains a common therapeutic option in the pharmacological control of heart rate in patients with atrial fibrillation, endorsed in current guidelines with the same level of evidence than betablockers in patients with and without heart failure. Digoxin has a narrow therapeutic range and is influenced by drug-to-drug interactions, serum electrolyte concentrations, and renal function.

Conflicting data exist regarding adverse outcomes that are associated with digoxin use in patients with atrial fibrillation. It remains unclear whether the association between digoxin use and worse clinical outcome is causal or may be the result of confounding by differences in the characteristics of patents including age, comorbidities and treatment. Particularly in older patients with atrial fibrillation, who are frequently prescribed a multitude of agents for stroke prevention, treatment of cardiovascular disease and other comorbidities, use of digoxin should be cautious and instituted with assessment of drug concentrations.
\end{abstract}

\section{Introduction}

Long-term pharmacological rate control is the preferred management strategy for atrial fibrillation (AF), among elderly and comorbid patients. Data from the EORP-AF Pilot (EURObservational Research Programme Atrial Fibrillation), a contemporary ESC Registry including 3119 subjects from 9 members ESC Countries, indicate that, at 1-year follow-up, $76 \%$ of those patients planned for rate control at baseline were continued in a rate control strategy. Besides, a rate control approach was considered in $15.6 \%$ of those patients who had been candidates for a

Corresponding author: Maria Teresa La Rovere, Department of Cardiology, Istituti Clinici Scientifici Maugeri, IRCCS Montescano, 27040 Montescano (PV), Italy. Tel. +39.0385.247290 - Fax +39.0385.61386.

E-mail: mariateresa.larovere@icsmaugeri.it

Key words: Digoxin; atrial fibrillation; rate control; prognosis; age.

Received for publication: 24 April 2018

Accepted for publication: 11 May 2018

CCopyright M.T. La Rovere and E. Traversi, 2018

Tipografia PI-ME Editrice, Italy

Monaldi Archives for Chest Disease 2018; 88:954

doi: 10.4081/monaldi.2018.954

This article is distributed under the terms of the Creative Commons Attribution Noncommercial License (by-nc 4.0) which permits any noncommercial use, distribution, and reproduction in any medium, provided the original author(s) and source are credited. rhythm control management strategy at baseline [1]. Patients aged $\geq 75$ years of age accounted for $45 \%$ and $21 \%$ of patients assigned to a rate or rhythm control strategy, respectively [2]. Beta-blockers were the most commonly used rate agents, followed by digoxin [2].

The frequent use of digoxin in patients with AF, particularly in elderly patients, is confirmed in the ROCKET AF trial that included over 14,000 patients with a mean age of 73 years [3]. Digoxin was prescribed in $37 \%$ of patients at baseline and $41 \%$ of patients were exposed to digoxin during the trial.

Indeed, the more recent European Guidelines [4] endorse the use of digoxin in AF, giving digoxin the same Class I, Level B indication than beta-blockers both in patients with and without heart failure. However, some controversies do exist on its safety. Digoxin has a narrow therapeutic range and digoxin toxicity is responsible for the third higher hospitalization rate for adverse drug events in elderly patients [5]. Whether the safety issue relate to the drug or to the type of patient (i.e., presence of comorbidities) treated with digoxin is unclear. At variance with heart failure, in AF there are no RCTs, and the conflicting evidence relating digoxin use and mortality is mainly deriving from post$h o c$ analyses and administrative data.

\section{Conflicting evidence on the association of digoxin use and mortality}

\section{Post-hoc analyses}

Two different post-hoc analysis of the AFFIRM trial yielding opposite conclusions, provide the emblematic representation of the conflicting evidence regarding digoxin use and mortality in AF [6,7]. In one study, Whitbeck et al. [6] found an association with digoxin use and an increase in all-cause mortality [HR 1.41, 95\% CI 1.19-1.67, $\mathrm{p}=0.001$ ] after controlling for clinical and demographic variables, as well as propensity score. By contrast, Gheorghiade et al. [7] revealed no association with digoxin and no increase in all-cause mortality [HR 1.06, 95\% CI $0.83-1.37, \mathrm{p}=0.67$ ] in a propensity-matched analysis. Differences in the statistical methods that have been adopted to analyze digoxin use can explain the divergent results [8]. In the Whitbeck's et al. [6] study, digoxin use was assessed at baseline and during followup, while in the Gheorghiade's et al. study [7], digoxin use was assessed at a fixed time point only, at the time of the baseline AFFIRM evaluation. With the first method, an indication bias is likely to be incurred in, as the analysis accounts for changes in digoxin use over time that might be related to changes in clinical status (i.e. heart failure) that could lead per se to the event (physicians tend to use digoxin in sicker patients). Vice versa, the second method can introduce a selection bias as a significant number of patients are excluded if data are missing at the fixed time point.

Finally, a third post-hoc analysis from the same AFFIRM trial even found that digoxin use was associated with improved survival in those patients with a markedly depressed left ventricular function $(<30 \%)[9]$. 
It is critical to note that, in the AFFIRM trial, high serum concentration of digoxin $(>1.0 \mathrm{ng} / \mathrm{mL})$ were encouraged to achieve the target heart rate for rate control. With the lower dosages prescribed in the RACE II (Rate Control Efficacy in Permanent AF: A Comparison between Lenient versus Strict rate Control II) trial, digoxin use was not associated with an impaired outcome [10]. By contrast, the largest post-hoc analysis from the ROCKET AF trial [3] showed an increased risk of all-cause mortality for digoxin treatment after adjustment for baseline variables.

\section{Registry data}

Observational studies and nationwide registries may be of utility for evaluating the association between digoxin and mortality in AF. However, similarly to post-hoc analyses, observational data are also conflicting in their results. It is worth noting that observational analyses are particularly prone to be confounded by the fact that sicker patients tend to receive digoxin more often and statistical adjustments cannot mitigate completely profound differences in baseline characteristics.

The TREAT-AF (The Retrospective Evaluation and Assessment of Therapies in AF) study [11] evaluated 122,465 patients with newly diagnosed, non valvular AF and seen within 90 days in the outpatient care setting of the U.S. Department of Veterans Affairs healthcare system. The mean age was $72.1 \pm 10.3$ years. Compared with nonrecipients, digoxin recipients were of similar age but had a higher prevalence of heart failure and receipt of other treatment drugs. Digoxin use was independently associated with mortality after multivariate adjustment [HR: 1.26, 95\% CI: 1.23-1.29, p<0.001]. The risk of death was not modified by age, sex, heart failure, kidney function, or concomitant use of beta-blockers, amiodarone, or warfarin.

The ORBIT-AF (Outcome Registry for Better Informed Treatment of Atrial Fibrillation), a US prospective outpatient registry specifically aimed to assess the use of digoxin and its association with outcome, provides a clinically detailed look at the patterns of digoxin use in patients with existing AF [12]. Medication use was collected prospectively at each study visit, including a field specific for digoxin. Among 9619 patients with AF and serial follow-up every 6 months for up to 3 years, 2267 (23.6\%) received digoxin at study enrollment, 681 (7.1\%) were initiated on digoxin during follow-up, and 6671 (69.4\%) were never prescribed digoxin. Overall $15 \%$ of ORBIT-AF patients either initiated or discontinued the drug, suggesting that use was dynamic. Prevalent digoxin use at registry enrollment was not associated with subsequent onset of symptoms, hospitalization, or mortality (in patients with HF, adjusted HR for death: 1.04; without HF, HR: 1.22). Incident digoxin use during follow-up was not associated with subsequent death in patients with HF (propensity adjusted HR: 1.05), but was associated with subsequent death in those without HF (propensity adjusted HR: 1.99).

The ATRIA-CVRN (AnTicoagulation and Risk factors In Atrial fibrillation-Cardiovascular Research Network) Study evaluated the independent association between newly initiated digoxin and the risk of death and hospitalization in a large, community-based cohort of adults with newly diagnosed AF and no documented heart failure [13]. In this analysis, involving 14,787 age, sex and high-dimensional propensity score-matched adults (mean age of the whole population $71.7 \pm 11.3$ years) digoxin use was independently associated with a $71 \%$ higher risk of death and a $63 \%$ higher risk of hospitalization. Among digoxin user who did have serum digoxin concentration measured, levels were significantly higher among those who died compared with those who did not die. However, it is worth to underscore that approximately $30 \%$ of the digoxin users never had a serum digoxin concentration measured and an additional $27 \%$ only had it measured once.

\section{Meta-analyses}

In an attempt to settle the uncertainty over the association of digoxin with adverse outcome, two systematic reviews including all studies published from 1993 to 2014 [14] and from 1960 to 2014 [15] have been undertaken. Remarkably, also the two meta-analyses were divergent in their findings. In one [14], based on 19 studies comprising more than 300,000 patients, digoxin therapy was associated with an increased mortality, particularly in those treated for AF and without proper serum level control. The second meta-analysis [15], included 52 observational studies and controlled trials comprising 621,845 patients, and showed that digoxin use has a neutral effect on mortality and reduces hospitalizations. These results lend the Authors to suggest that digoxin should continue to be considered as a treatment option to achieve control of heart rate in those with AF and to avoid hospital admissions in patients with heart failure.

A very large nationwide population-based study - not included in the above systematic reviews - investigated the risk of mortality in patients without rate-control treatments compared with those who received different rate-control drugs [16]. Patients with combinations of rate control drugs were excluded. After adjustment for baseline differences, the risk of mortality was lower in patients receiving $\beta$-blockers (adjusted HR=0.76; 95\% confidence interval=0.74-0.78) and calcium channel blockers (adjusted $\mathrm{HR}=0.93 ; 95 \%$ confidence interval $=0.90-0.96$ ) compared with those who did not receive rate-control medications. On the contrary, the digoxin group had a higher risk of mortality with an adjusted HR of 1.12 (95\% confidence interval $=1.10-1.14$ ).

\section{Digoxin and the elderly patient with AF}

Polypharmacy (defined as the use of 5 or more regular medications) in common in older adults. Older AF patients are frequently prescribed a multitude of agents for stroke prevention, management of the arrhythmia, treatment of cardiovascular disease and other comorbidities. In these patients, the prevalence of polypharmacy has been reported up to $94.8 \%$ [17]. Although prescribed and over-the-counter medications may improve a wide range of health problems, they also may cause or contribute to harm, especially in older adults. Digoxin has a complex pharmacokinetic profile, a narrow therapeutic range and multiple drug interactions that may increase the risk of digoxin toxicity. Thus, the assessment of patient-specific factors that can influence the dose-effect relationship such age, liver and renal function, body habitus, comorbid conditions and medications is of utmost importance when prescribing digoxin in the elderly.

\section{Conclusions and clinical implications}

Although beta-blockers are the most commonly used rate control agents in AF, digoxin use remains high in the contemporary treatment of AF. Guidelines do not assist clinicians in choosing first-line and subsequent therapy other than suggesting that the choice of medication should be individualized, depending on the presence of ongoing symptoms. In the ongoing RATE-AF (Rate control Therapy Evaluation in permanent Atrial Fibrillation) study [18], digoxin and bisoprolol will be compared head-to head as the initial rate control agent in patients with permanent AF with NYHA class II and above to assess the effect of therapy on patient-reported quality of life. While the trial will also evaluate the long-term impact on cardiac function, it will not have the power to identify differences in clinical events. 
Whether digoxin is associated with an increased mortality risk remains unclear. An argument is made that patients prescribed digoxin are sicker and that is why they have a higher mortality in observational studies. Because AF is one of the evolving epidemics in cardiovascular medicine, assessing the association between digoxin use adverse outcomes is of utmost importance. As an RCT is unlikely to be performed, a pragmatic trial of real-world contemporary digoxin use should be a key priority.

Particularly in older patients, the effect of digoxin on outcome may depend on the type of patients and the dosage used. Cautious use of lowdose digoxin is suggested in older patients, in patients with renal insufficiency and in patients with concomitant use of other drugs that could raise digoxin concentrations. Although not commonly performed in community practice, assessment of the digoxin plasma concentration can help in dose finding and modulation during the course of the disease.

\section{References}

1. Lip GY, Laroche C, Ioachim PM, et al. Prognosis and treatment of atrial fibrillation patients by European cardiologists: one year follow-up of the EURObservational Research Programme-Atrial Fibrillation General Registry Pilot Phase (EORP-AF Pilot registry). Eur Heart J 2014;35:3365-76.

2. Purmah Y, Proietti M, Laroche C, et al. Rate vs. rhythm control and adverse outcomes among European patients with atrial fibrillation. Europace 2017 Feb 4. doi: 10.1093/europace/euw421. [Epub ahead of print].

3. Washam JB, Stevens SR, Lokhnygina Y, et al. Digoxin use in patients with atrial fibrillation and adverse cardiovascular outcomes: a retrospective analysis of the rivaroxaban once daily oral direct factor $\mathrm{Xa}$ inhibition compared with vitamin $\mathrm{K}$ antagonism for prevention of stroke and embolism trial in atrial fibrillation (ROCKET AF). Lancet 2015;385:2363-70.

4. Kirchhof P, Benussi S, Kotecha D, et al. 2016 ESC Guidelines for the management of atrial fibrillation developed in collaboration with EACTS. Europace 2016;18:1609-78.

5. Budnitz DS, Lovegrove MC, Shehab N, Richards CL. Emergency hospitalizations for adverse drug events in older Americans. N Engl J Med 2011;365:2002-12.
6. Whitbeck MG, Charnigo RJ, Khairy P, et al. Increased mortality among patients taking digoxin-analysis from the AFFIRM study. Eur Heart J 2013;34:1481-8.

7. Gheorghiade M, Fonarow GC, van Veldhuisen DJ, et al. Lack of evidence of increased mortality among patients with atrial fibrillation taking digoxin: findings from post hoc propensitymatched analysis of the AFFIRM trial. Eur Heart J 2013;34:1489-97.

8. Murphy SA. When "digoxin use" is not the same as "digoxin use": lessons from the AFFIRM trial. Eur Heart J 2013;34:1465-7.

9. Patel NJ, Hoosien M, Deshmukh A, et al. Digoxin significantly improves all-cause mortality in atrial fibrillation patients with severely reduced left ventricular systolic function. Int $\mathrm{J}$ Cardiol 2013;169:e84-6.

10. Mulder BA, Van Veldhuisen DJ, Crijns HJ, et al. Digoxin in patients with permanent atrial fibrillation: data from the RACE II study. Heart Rhythm 2014;11:1543-50.

11. Turakhia MP, Santangeli P, Winkelmayer WC, et al. Increased mortality associated with digoxin in contemporary patients with atrial fibrillation: findings from the TREAT-AF study. J Am Coll Cardiol 2014;64:660-8.

12. Allen LA, Fonarow GC, Simon DN, et al. Digoxin use and subsequent outcomes among patients in a contemporary atrial fibrillation cohort. J Am Coll Cardiol 2015;65:2691-8.

13. Freeman JV, Reynolds K, Fang M, et al. Digoxin and risk of death in adults with atrial fibrillation: the ATRIA-CVRN study. Circ Arrhythm Electrophysiol 2015;8:49-58.

14. Vamos M, Erath JW, Hohnloser SH. Digoxin-associated mortality: a systematic review and meta-analysis of the literature. Eur Heart J 2015;36:1831-8

15. Ziff OJ, Lane DA, Samra M, et al. Safety and efficacy of digoxin: systematic review and meta-analysis of observational and controlled trial data. BMJ 2015;51:h4451.

16. Chao TF, Liu CJ, Tuan TC, et al. Rate-control treatment and mortality in atrial fibrillation. Circulation 2015;132:1604-12.

17. Wang Y, Singh S, Bajorek B. Old age, high risk medication, polypharmacy: a 'trilogy' of risks in older patients with atrial fibrillation. Pharm Pract 2016;14:706.

18. Kotecha D, Calvert M, Deeks JJ, et al. A review of rate control in atrial fibrillation, and the rationale and protocol for the RATE-AF trial. BMJ Open 2017;7:e015099. 